\title{
ROTUNDITY AND SMOOTHNESS IN CONJUGATE SPACES ${ }^{1}$
}

\author{
M. A. SMITH
}

\begin{abstract}
It is shown that the James space $J$ admits an equivalent norm such that its third conjugate space is rotund.
\end{abstract}

A Banach space $B$ is said to be rotund if $\|x+y\|<2$ whenever $x$, $y \in S(B) \equiv\{z \in B:\|z\|=1\}$ and $x \neq y$. A Banach space $B$ with conjugate space $B^{*}$ is said to be smooth if for every $x \in S(B)$ there exists a unique $x^{*} \in S\left(B^{*}\right)$ such that $x^{*}(x)=1$. Note that $B$ is smooth if $B^{*}$ is rotund, and $B$ is rotund if $B^{*}$ is smooth.

The purpose of this note is to show the existence of a nonreflexive Banach space with rotund third conjugate space, a matter of interest raised by Rainwater [8] and Diestel [3, p. 43]. Specifically, we show that the quasi-reflexive space $J$ of James [6] admits an equivalent norm such that its third conjugate space is rotund.

For a Banach space $B$, we denote its successive conjugate spaces by $B^{*}$, $B^{* *}, B^{* * *}$, and $B^{(4)}$. We denote the canonical isometric embeddings of $B, B^{*}$, and $B^{* *}$ into $B^{* *}, B^{* * *}$, and $B^{(4)}$ by $Q_{0}, Q_{1}$, and $Q_{2}$ respectively. When no ambiguity exists, we shall omit these embecidings and write, for example, $x \in B^{* *}$ instead of $Q_{0}(x) \in B^{* *}$ for $x \in B$. Recall that we may write $B^{* * *}=B^{*} \oplus B^{\perp}$, and that $\left\|x^{*}\right\| \leqslant\left\|x^{*}+x^{\perp}\right\|$ for all $x^{*} \in B^{*}$ and $x^{\perp} \in$ $B^{\perp}$.

For an arbitrary nonreflexive Banach space $B$, Dixmier [4] has shown that $B^{(4)}$ is not rotund. Phelps, Day [1, p. 70], Giles [5], and Rainwater [8] have independently strengthened Dixmier's result by showing that $B^{* * *}$ is not smooth for $B$ nonreflexive. Combining these results, we have that if $B$ is nonreflexive, then the $n$th conjugate space $B^{(n)}$, for $n \geqslant 4$, is neither rotund nor smooth. On the positive side, from the work of Zizler [11], we have that if $B^{*}$ is separable, then $B$ admits an equivalent norm such that $B$ and $B^{*}$ are both rotund and smooth, and $B^{* *}$ is rotund. In [9], we established the existence of a smooth, nonreflexive second conjugate space. In this paper, we complete the picture of rotundity and smoothness in conjugate spaces by showing the existence of a rotund, nonreflexive third conjugate space. Combining the proof of our result with a result of Zizler [11, p. 200], we have the best possible result that there exists a nonreflexive Banach space $B$ such that

Received by the editors June 17, 1976 and, in revised form, August 23, 1976.

AMS (MOS) subject classifications (1970). Primary 46B10, 46B99.

${ }^{1}$ Research supported in part by a Lake Forest College Summer Research Grant. 
$B, B^{*}$, and $B^{* *}$ are both rotund and smooth, and $B^{* * *}$ is rotund.

We shall need the following strengthenings of rotundity.

A Banach space $B$ is said to be locally uniformly rotund if whenever $x \in S(B)$ and $\left\{x_{n}\right\}$ is a sequence in $B$ such that $\left\|x_{n}\right\| \rightarrow 1$ and $\left\|x+x_{n}\right\| \rightarrow 2$, then $x_{n} \rightarrow x$.

A conjugate space $B^{*}$ is said to be weak ${ }^{*}$ uniformly rotund if whenever $\left\{x_{n}^{*}\right\}$ and $\left\{y_{n}^{*}\right\}$ are sequences in $B^{*}$ such that $\left\|x_{n}^{*}\right\| \rightarrow 1,\left\|y_{n}^{*}\right\| \rightarrow 1$, and $\left\|x_{n}^{*}+y_{n}^{*}\right\| \rightarrow 2$, then $x_{n}^{*}-y_{n}^{*} \rightarrow 0$ weak*

The following result is due to Lindenstrauss and Rosenthal [7] and Dean [2].

The Principle of Local Reflexivity. Let $Z$ in $B^{* *}$ and $F$ in $B^{*}$ be finite dimensional subspaces, and let $0<\varepsilon<1$ be arbitrary. Then there exists a linear map $T: Z \rightarrow B$ such that

(a) $T(z)=z$ for all $z \in Z \cap B$,

(b) $f(T(z))=z(f)$ for all $z \in Z$ and $f \in F$,

(c) $(1-\varepsilon)\|z\| \leqslant\|T(z)\| \leqslant(1+\varepsilon)\|z\|$ for all $z \in Z$.

We follow the lead of Sullivan [10] in using the Principle of Local Reflexivity to obtain geometric results.

THEOREM. The James space $J$ admits an equivalent norm such that its third conjugate space is rotund.

Proof. The crucial properties of $J$ that we need are that $J$ is separable and quasi-reflexive of order one. Since $J^{*}$ is separable, $J$ admits an equivalent norm such that its conjugate is both weak* uniformly rotund and locally uniformly rotund (see $[11$, p. 200]). Let $(B,\|\cdot\|)$ denote $J$ under such a norm. Write $B^{* * *}=B^{*} \oplus\left[b^{* * *}\right]$ where $\left[b^{* * *}\right]$ denotes the linear span of $\left\{b^{* * *}\right\}$ and $b^{* * *} \in B^{\perp}$.

We claim that $B^{* * *}$ is rotund. To show this, suppose $x^{* * *}$ and $y^{* * *}$ are norm-1 elements in $B^{* * *}$ such that $\left\|x^{* * *}+y^{* * *}\right\|=2$. Write $x^{* * *}=x^{*}+$ $\alpha b^{* * *}$ and $y^{* * *}=y^{*}+\beta b^{* * *}$ where $x^{*}$ and $y^{*}$ are in $B^{*}$ and $\alpha$ and $\beta$ are scalars. If $x^{*} \neq y^{*}$, then there exists $x \in S(B)$ such that $\left(x^{*}-y^{*}\right)(x) \neq 0$. By the Principle of Local Reflexivity, there exists a sequence of linear maps $T_{n}: \operatorname{span}\left\{x^{* * *}, y^{* * *}\right\} \rightarrow B^{*}$ such that

$$
Q_{0}(x)\left(T_{n}\left(x^{* * *}-y^{* * *}\right)\right)=\left(x^{* * *}-y^{* * *}\right)(x)=\left(x^{*}-y^{*}\right)(x)
$$

and

$$
\left(1-\varepsilon_{n}\right)\|z\| \leqslant\left\|T_{n}(z)\right\| \leqslant\left(1+\varepsilon_{n}\right)\|z\|
$$

for all $z \in \operatorname{span}\left\{x^{* * *}, y^{* * *}\right\}$, where $\left\{\varepsilon_{n}\right\}$ is a positive sequence decreasing to zero. Let $x_{n}^{*}=T_{n}\left(x^{* * *}\right)$ and $y_{n}^{*}=T_{n}\left(y^{* * *}\right)$. Then we have $\left\|x_{n}^{*}\right\| \rightarrow 1,\left\|y_{n}^{*}\right\|$ $\rightarrow 1$, and $\left\|x_{n}^{*}+y_{n}^{*}\right\| \rightarrow 2$. But $\left(x_{n}^{*}-y_{n}^{*}\right)(x)=\left(x^{*}-y^{*}\right)(x) \neq 0$, contradicting the fact that $B^{*}$ is weak ${ }^{*}$ uniformly rotund. Therefore $x^{*}=y^{*}$. Choose $x^{(4)} \in S\left(B^{(4)}\right)$ such that $x^{(4)}\left(x^{* * *}\right)=x^{(4)}\left(y^{* * *}\right)=1$. Then we have

$$
0=x^{(4)}\left(x^{* * *}-y^{* * *}\right)=(\alpha-\beta) x^{(4)}\left(b^{* * *}\right) \text {. }
$$


If $x^{(4)}\left(b^{* * *}\right) \neq 0$, then $\alpha=\beta$, and hence $x^{* * *}=y^{* * *}$. If $x^{(4)}\left(b^{* * *}\right)=0$, then $x^{(4)}\left(x^{*}\right)=x^{(4)}\left(x^{*}+\alpha b^{* * *}\right)=x^{(4)}\left(x^{* * *}\right)=1$.

From this, and since $\left\|x^{*}\right\| \leqslant\left\|x^{*}+\alpha b^{* * *}\right\|=1$, it follows that $\left\|x^{*}\right\|=1$ and $\left\|x^{*}+x^{* * *}\right\|=2$. If $x^{* * *} \neq x^{*}$, then by the Principle of Local Reflexivity, as in Lemma 7 of [10], there exists a sequence of linear maps $T_{n}$ : $\operatorname{span}\left\{Q_{1}\left(x^{*}\right), x^{* * *}\right\} \rightarrow B^{*}$ such that $T_{n}\left(Q_{1}\left(x^{*}\right)\right)=x^{*}$ for each $n$ and

$$
\left(1-\varepsilon_{n}\right)\|z\| \leqslant\left\|T_{n}(z)\right\| \leqslant\left(1+\varepsilon_{n}\right)\|z\|
$$

for all $z \in \operatorname{span}\left\{Q_{1}\left(x^{*}\right), x^{* * *}\right\}$, where $\left\{\varepsilon_{n}\right\}$ is a positive sequence decreasing to zero. Let $x_{n}^{*}=T_{n}\left(x^{* * *}\right)$. Then we have $\left\|x^{*}\right\|=1,\left\|x_{n}^{*}\right\| \rightarrow 1$, and $\| x^{*}+$ $x_{n}^{*} \| \rightarrow 2$. But $\left\|x^{*}-x_{n}^{*}\right\| \geqslant\left(1-\varepsilon_{n}\right)\left\|x^{*}-x^{* * *}\right\|$, contradicting the fact that $B^{*}$ is locally uniformly rotund. Therefore $x^{* * *}=x^{*}$. Similarly, it follows that $y^{* * *}=x^{*}$, and hence $x^{* * *}=y^{* * *}$. This completes the proof.

AdDed Note. After this paper was submitted, Professor Daniel Amir pointed out that the theorem can also be proved using the fact that the unit ball of $B^{*}$ is weak* sequentially dense in the unit ball of $B^{* * *}$, since $B^{* *}$ is separable, and without using the Principle of Local Reflexivity.

\section{REFERENCES}

1. M. M. Day, Normed linear spaces, 3rd ed., Ergebnisse Math. Grenzgebiete, Band, 21, Springer-Verlag, New York, 1973. MR 49 \#9588.

2. D. W. Dean, The equation $L\left(E, X^{* *}\right)=L(E, X)^{* *}$ and the principle of local reflexivity, Proc. Amer. Math. Soc. 40 (1973), 146-148. MR 48 \#2735.

3. J. Diestel, Geometry of Banach spaces-Selected topics, Lecture Notes in Math., vol. 485, Springer-Verlag, Berlin and New York, 1975.

4. J. Dixmier, Sur un théorème de Banach, Duke Math. J. 15 (1948), 1057-1071. MR $10,306$.

5. J. R. Giles, $A$ non-reflexive Banach space has non-smooth third conjugate space, Canad. Math. Bull. 17 (1974), 117-119. MR 50 \# 5429.

6. R. C. James, A non-reflexive Banach space isometric with its second conjugate space, Proc. Nat. Acad. Sci. U.S.A. 37 (1951), 174-177. MR 13, 356.

7. J. Lindenstrauss and H. P. Rosenthal, The $\mathcal{E}_{p}$ spaces, Israel J. Math. 7 (1969), 325-349. MR 42 \# 5012.

8. J. Rainwater, A non-reflexive Banach space has non-smooth third conjugate space (unpublished).

9. M. A. Smith, A smooth, non-reflexive second conjugate space, Bull. Austral. Math. Soc. 15 (1976), 129-131.

10. F. Sullivan, Geometrical properties determined by the higher duals of a Banach space (preprint).

11. V. Zizler, Some notes on various rotundity and smoothness properties of separable Banach spaces, Comment. Math. Univ. Carolinae 10 (1969), 195-206. MR 39 \#401.

Department of Mathematics, Lake Forest College, Lake Forest, Illinois 60045 